\title{
Heat Transfer in Microchannels with Upstream-Downstream Regions Coupling and Wall Conjugation Effects
}

\author{
Diego C. Knupp , Renato M. Cotta \& Carolina P. Naveira-Cotta
}

To cite this article: Diego C. Knupp, Renato M. Cotta \& Carolina P. Naveira-Cotta (2013) Heat Transfer in Microchannels with Upstream-Downstream Regions Coupling and Wall Conjugation Effects, Numerical Heat Transfer, Part B: Fundamentals, 64:5, 365-387, DOI: 10.1080/10407790.2013.810535

To link to this article: https://doi.org/10.1080/10407790.2013.810535

\section{曲 Published online: 17 Sep 2013.}

Submit your article to this journal $\longleftarrow$

Џ Article views: 96

Citing articles: 13 View citing articles $[3$ 


\title{
HEAT TRANSFER IN MICROCHANNELS WITH UPSTREAM-DOWNSTREAM REGIONS COUPLING AND WALL CONJUGATION EFFECTS
}

\author{
Diego C. Knupp, Renato M. Cotta, and \\ Carolina P. Naveira-Cotta \\ Laboratory of Transmission and Technology of Heat, \\ Laboratory of Microfluidics and Micro-systems, Mechanical Engineering \\ Department, Universidade Federal do Rio de Janeiro, Rio de Janeiro, Brazil
}

Heat transfer in microchannels is analyzed, including the coupling between the regions upstream and downstream of the heat transfer section and taking into account the wall conjugation and axial diffusion effects which are often of relevance in microchannels. The methodology is based on a recently proposed single-domain formulation for modeling the heat transfer phenomena simultaneously at the fluid stream and the channel walls, and applying the generalized integral transform technique (GITT) to find a hybrid numerical-analytical solution to the unified partial differential energy equation. The proposed mathematical model involves coefficients represented as space-dependent functions, with abrupt transitions at the fluid-wall interfaces, which carry the information concerning the transition of the two domains, unifying the model into a single-domain formulation with variable coefficients. Convergence of the proposed eigenfunction expansions is thoroughly investigated and the physical analysis is focused on the effects of the coupling between the downstream and the upstream flow regions.

\section{INTRODUCTION}

The conception and design of thermal microsystems has been a quite active research area in heat transfer, initially just connected with the cooling of electronic components, but in more recent years also of crucial importance in various applications related to microsystems that require micro-heat exchangers, microheat spreaders, and microreactors $[1,2]$. Within the last decade, a few review works have identified discrepancies between micro-scale experimental results for friction factors and Nusselt numbers within microchannels from different contributions, not only among themselves, but also against simulations and macroscale correlations [3, 4]. Although such discrepancies, especially among different sets of experimental results, can be in part explained by the inherent experimental difficulties at the microscale,

Received 27 January 2013; accepted 8 May 2013.

The authors would like to acknowledge financial support provided by the federal and state Brazilian research sponsoring agencies, CNPq and FAPERJ.

Address correspondence to Renato M. Cotta, Mechanical Engineering Department, Universidade Federal do Rio de Janeiro, EE/COPPE, C.P. 68503, Rio de Janeiro, RJ 21945-970, Brazil. E-mail: cotta@mecanica.coppe.ufrj.br 


\begin{tabular}{|c|c|c|c|}
\hline \multicolumn{4}{|c|}{ NOMENCLATURE } \\
\hline $\mathrm{Bi}$ & Biot number & Greek lette & \\
\hline$h_{e}$ & heat transfer coefficient at the & $\alpha$ & thermal diffusivity \\
\hline & channel external wall & $\beta, \alpha, \lambda, v$ & eigenvalues corresponding to $\xi, \zeta, \Omega$ \\
\hline$K$ & dimensionless thermal conductivity & & and $\phi$, respectively \\
\hline$k$ & thermal conductivity & $\zeta$ & eigenfunction of the insulated \\
\hline$L_{e}$ & distance from the channel centerline & & region eigenvalue problem \\
\hline & to the external face of the channel & $\theta$ & dimensionless temperature field \\
\hline & wall & $\xi$ & eigenfunction of the heat exchange \\
\hline$L_{f}$ & channel height & & section eigenvalue problem \\
\hline$L_{w}$ & channel width & $\phi$ & eigenfunction of the auxiliary \\
\hline M & $\begin{array}{l}\text { truncation order of the auxiliary } \\
\text { problems }\end{array}$ & & $\begin{array}{l}\text { problem corresponding to insulated } \\
\text { region eigenvalue problem }\end{array}$ \\
\hline $\mathrm{N}$ & $\begin{array}{l}\text { truncation order of the temperature } \\
\text { field expansions }\end{array}$ & $\Omega$ & $\begin{array}{l}\text { eigenfunction of the auxiliary } \\
\text { problem corresponding to heat }\end{array}$ \\
\hline $\mathrm{Nu}$ & Nusselt number & & exchange section eigenvalue \\
\hline $\mathrm{Pe}$ & Péclet number & & problem \\
\hline$T$ & temperature field & & \\
\hline$U$ & $\begin{array}{l}\text { dimensionless fully developed } \\
\text { velocity profile }\end{array}$ & $\begin{array}{l}\text { Subscripts } \\
\text { ad }\end{array}$ & Id Superscripts \\
\hline$u$ & flow velocity & & upstream region \\
\hline$w$ & heat capacity & $i, m, n$ & order of eigenquantities \\
\hline$Y$ & $\begin{array}{l}\text { dimensionless transversal } \\
\text { coordinate }\end{array}$ & - & $\begin{array}{l}\text { integral transform } \\
\text { normalized eigenfunction }\end{array}$ \\
\hline$y$ & transversal coordinate & $f$ & fluid \\
\hline$Z$ & dimensionless longitudinal & $\begin{array}{l}s \\
\text { in }\end{array}$ & $\begin{array}{l}\text { solid } \\
\text { channel inlet }\end{array}$ \\
\hline$z$ & $\begin{array}{l}\text { coordinate } \\
\text { longitudinal coordinate }\end{array}$ & $\infty$ & external environment \\
\hline
\end{tabular}

some reported discrepant experimental, simulated, and correlation results may be a consequence of neglecting phenomena that are usually not accounted for at the macroscale, but that may be significant in microscale heat transfer. A number of works are then still devoted to building and revisiting reliable models and solution methodologies capable of describing the physical phenomena that take place in such microscale problems. For instance, the consideration of slip flow in opposition to the classical no-slip condition for gas flow, the inclusion of terms related to viscous dissipation, wall conjugation, axial diffusion, and corrugated walls effects, which are often neglected in macroscale problems, illustrate some of the efforts to include effects that are not commonly accounted for in macroscale situations [5-13]. Nunes et al. [13] presented some experimental and theoretical results showing the importance of considering heat conduction along metallic microchannel walls, leading to a conjugated problem that yields results in better agreement with the reported experimental data.

Recently, the reformulation of conjugated conduction-convection problems as a single-region model has been proposed, accounting for the local heat transfer at both the fluid flow and the channel wall regions [14]. By making use of coefficients represented as space-variable functions, with abrupt transitions occurring at the fluid-wall interfaces, the mathematical model incorporates the information concerning the two original domains of the problem. In the solution of the proposed single-domain mathematical model, the generalized integral transform technique (GITT) [15-20] is 
employed. This approach is based on extending the classical integral transform method, making it sufficiently flexible to handle problems that are not a priori transformable, such as in the case of problems with arbitrarily space-dependent coefficients. In [14], a fairly simple test case was selected, for which an exact solution was obtainable for comparison purposes, neglecting axial conduction within both the fluid and the walls. The excellent agreement between the results obtained with the proposed methodology and the exact solution motivated the extension of this novel approach to the solution of more involved conjugated heat transfer problems. Thus, in [21] such developments progressed toward the flow in microchannels, further developing and validating this single-domain formulation in dealing with axial and transversal heat diffusion, both at the channel walls and in the fluid stream.

In the present work we advance the methodology initially proposed in [14, 21] in order to take into account the heat conduction to the usually assumed adiabatic region, upstream of the heat exchange section, which may however play an important role in the heat transfer problem for low Péclet numbers, typical of applications with microchannels at low Reynolds numbers, as pointed out in [13]. As a test case, an actual configuration is considered consisting of a rectangular microchannel etched on a nanocomposite substrate made of polyester resin and alumina nanoparticles [22] and the effects of axial diffusion in both the wall and fluid conjugated regions are investigated, considering the effect of heat transfer to the supposedly adiabatic region upstream of the heat exchange section.

The GITT is again employed in the direct integral transformation of the merged fluid-solid energy equations, as well as on the solution of the associated eigenvalue problems with space-variable coefficients [22, 23], written for both the upstream and downstream regions. Numerical results are then employed to illustrate the convergence behavior of the single-domain eigenfunction expansions and to allow for a physical analysis on the influence of heat transfer to the upstream region in terms of the governing parameters. The obtained error-controlled numerical results are then employed for critical comparisons with a commercial computational fluid dynamics (CFD) software system.

\section{PROBLEM FORMULATION AND SOLUTION METHODOLOGY}

Figure 1 shows a schematic representation of the problem under consideration. We consider steady-state laminar incompressible internal flow of a Newtonian fluid inside a rectangular channel with height and width given by $L_{f}$ and $L_{w}$, respectively, with $L_{w} \gg L_{f}$ so that it can be approximately described by a flow between parallel plates. The channel walls are considered to participate on the heat transfer problem through both transversal and axial heat conduction. The fluid flows with a fully developed velocity profile $u_{f}(y)$, and with uniform inlet temperature $T_{\text {in }}$. The flow is assumed to be hydrodynamically developed but thermally developing, with negligible viscous dissipation and temperature-independent physical properties. The external face of the microchannel wall exchanges heat with the surrounding environment by convection with a heat transfer coefficient, $h_{e}$. We consider that the microchannel heat exchange section, $0 \leq z \leq z_{\infty}$, also exchanges heat with the transversally insulated upstream region, $-z_{\mathrm{ad}, \infty} \leq z \leq 0$. Making use of the problem symmetry at $y=0$, the formulation of the conjugated problem as a single-region 


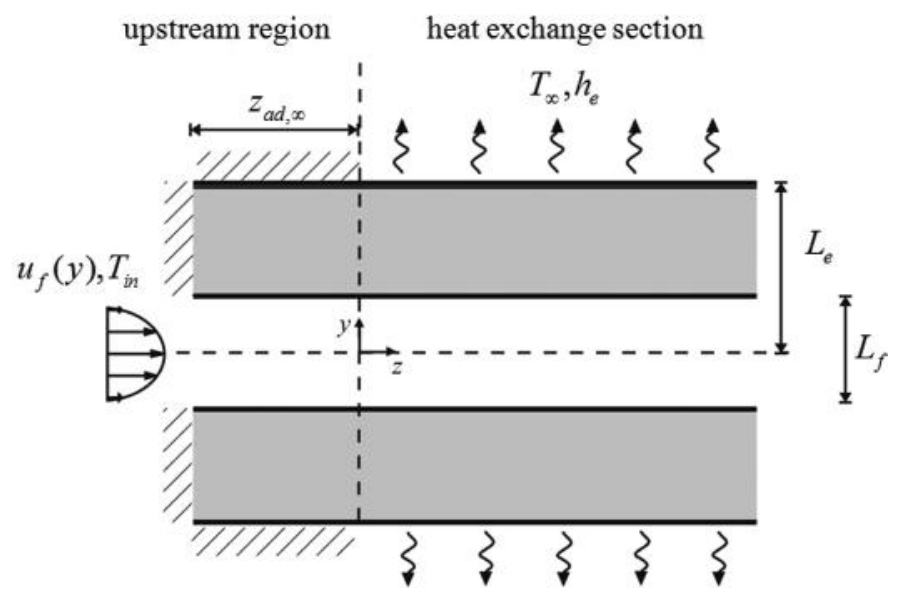

Figure 1. Schematic representation of the conjugate heat transfer problem in a microchannel.

model is achieved by making use of space-variable coefficients with abrupt transitions at the fluid-solid wall interface, as follows.

Heat exchange section:

$$
\begin{gathered}
u(y) w_{f} \frac{\partial T(y, z)}{\partial z}=k(y) \frac{\partial^{2} T}{\partial z^{2}}+\frac{\partial}{\partial y}\left[k(y) \frac{\partial T}{\partial y}\right], \quad 0<y<L_{e}, \quad 0<z<z_{\infty} \\
T(y, 0)=T_{\mathrm{ad}}(y, 0),\left.\quad \frac{\partial T}{\partial z}\right|_{z=z_{\infty}}=0, \quad 0<y<L_{e} \\
\left.\frac{\partial T}{\partial y}\right|_{y=0}=0,\left.\quad k\left(L_{e}\right) \frac{\partial T}{\partial y}\right|_{y=L_{e}}+h_{e} T\left(L_{e}, z\right)=h_{e} T_{\infty}, \quad 0<z<z_{\infty}
\end{gathered}
$$

Insulated upstream region:

$$
\begin{gathered}
u(y) w_{f} \frac{\partial T_{\mathrm{ad}}(y, z)}{\partial z}=k(y) \frac{\partial^{2} T_{\mathrm{ad}}}{\partial z^{2}}+\frac{\partial}{\partial y}\left[k(y) \frac{\partial T_{\mathrm{ad}}}{\partial y}\right], \quad 0<y<L_{e}, \quad-z_{\mathrm{ad}, \infty}<z<0 \\
T_{\mathrm{ad}}\left(y,-z_{\mathrm{ad}, \infty}\right)=T_{\mathrm{in}},\left.\quad \frac{\partial T_{\mathrm{ad}}}{\partial z}\right|_{z=0}=\left.\frac{\partial T}{\partial z}\right|_{z=0}, \quad 0<y<L_{e} \quad(1 g, h) \\
\left.\frac{\partial T_{\mathrm{ad}}}{\partial y}\right|_{y=0}=0,\left.\quad \frac{\partial T_{\mathrm{ad}}}{\partial y}\right|_{y=L_{e}}=0, \quad-z_{\mathrm{ad}, \infty}<z<0
\end{gathered}
$$

with

$$
u(y)=\left\{\begin{array}{ll}
u_{f}(y) & \text { if } 0<y<L_{f} / 2 \\
0 & \text { if } L_{f} / 2<y<L_{e}
\end{array}, \quad k(y)=\left\{\begin{array}{ll}
k_{f} & \text { if } 0<y<L_{f} / 2 \\
k_{s} & \text { if } L_{f} / 2<y<L_{e}
\end{array} \quad(1 k, l)\right.\right.
$$


where $w_{f}$ is the fluid heat capacity, $k_{s}$ is the channel wall thermal conductivity, $k_{f}$ is the fluid thermal conductivity, and $u_{f}(y)$ is the parabolic velocity profile. The following dimensionless groups are adopted:

$$
\begin{aligned}
Z & =\frac{z / D_{h}}{\operatorname{Re} \operatorname{Pr}}=\frac{z}{D_{h} \operatorname{Pe}}, \quad Y=\frac{y}{L_{e}}, \quad U=\frac{u}{u_{\mathrm{av}}} \\
\theta & =\frac{T-T_{\infty}}{T_{\mathrm{in}}-T_{\infty}}, \quad K=\frac{k}{k_{f}}, \quad \operatorname{Re}=\frac{u_{\mathrm{av}} D_{h}}{\nu}, \\
\operatorname{Pr} & =\frac{\nu}{\alpha}, \quad \operatorname{Pe}=\operatorname{Re} \operatorname{Pr}=\frac{u_{\mathrm{av}} D_{h}}{\alpha}, \quad \alpha=\frac{k_{f}}{w_{f}}, \\
\mathrm{Bi} & =\frac{h_{e} L_{e}}{k\left(L_{e}\right)}, \quad \sigma=\frac{L_{e}}{L_{f}}
\end{aligned}
$$

where the hydraulic diameter is given by $D_{h}=2 L_{f}$. After making use of the groups given by Eqs. ( $2 \mathrm{a}-\mathrm{k})$, one obtains the dimensionless formulation, where we have incorporated a pseudo-transient term in order to solve the problem by means of the partial integral transformation scheme of the GITT [9, 24], yielding the following.

Heat exchange section:

$$
\begin{gathered}
\frac{\partial \theta(Y, Z, t)}{\partial t}=-U(Y) \frac{\partial \theta}{\partial Z}+\frac{K(Y)}{\mathrm{Pe}^{2}} \frac{\partial^{2} \theta}{\partial Z^{2}} \\
+\frac{4}{\sigma^{2}} \frac{\partial}{\partial Y}\left[K(Y) \frac{\partial \theta}{\partial Y}\right], \quad 0<Y<1,0<Z<Z_{\infty}, t>0 \\
\theta(Y, 0, t)=\theta_{\mathrm{ad}},\left.(Y, 0, t) \quad \frac{\partial \theta}{\partial Z}\right|_{Z=Z_{\infty}}=0, \quad 0<Y<1, t>0 \\
\left.\frac{\partial \theta}{\partial Y}\right|_{Y=0}=0,\left.\quad \frac{\partial \theta}{\partial Y}\right|_{Y=1}+\operatorname{Bi\theta }(1, Z, t)=0, \quad 0<Z<Z_{\infty}, t>0 \\
\theta(Y, Z, 0)=f(Z, Y), \quad 0<Y<1, \quad 0<Z<Z_{\infty}
\end{gathered}
$$

Insulated upstream region:

$$
\begin{gathered}
\frac{\partial \theta_{\mathrm{ad}}(Y, Z, t)}{\partial t}=-U(Y) \frac{\partial \theta_{\mathrm{ad}}}{\partial Z}+\frac{K(Y)}{\mathrm{Pe}^{2}} \frac{\partial^{2} \theta_{\mathrm{ad}}}{\partial Z^{2}}+\frac{4}{\sigma^{2}} \frac{\partial}{\partial Y}\left[K(Y) \frac{\partial \theta_{\mathrm{ad}}}{\partial Y}\right], \\
0<Y<1,-Z_{\mathrm{ad}, \infty}<Z<0, t>0 \\
\theta_{\mathrm{ad}}\left(Y,-Z_{\mathrm{ad}, \infty}, t\right)=1,\left.\quad \frac{\partial \theta_{\mathrm{ad}}}{\partial Z}\right|_{z=0}=\left.\frac{\partial \theta}{\partial Z}\right|_{z=0}, \quad 0<Y<1, t>0 \\
\left.\frac{\partial \theta_{\mathrm{ad}}}{\partial Y}\right|_{Y=0}=0,\left.\quad \frac{\partial \theta_{\mathrm{ad}}}{\partial Y}\right|_{Y=1}=0, \quad-Z_{\mathrm{ad}, \infty}<Z<0, t>0
\end{gathered}
$$




$$
\theta_{\mathrm{ad}}(Y, Z, 0)=f_{\mathrm{ad}}(Z, Y), \quad 0<Y<1, \quad-Z_{\mathrm{ad}, \infty}<Z<0
$$

with

$$
\begin{aligned}
& U(Y)= \begin{cases}U_{f}(Y) & \text { if } 0<Y<Y_{i}=L_{f} / 2 L_{e} \\
0 & \text { if } Y_{i}<Y<1\end{cases} \\
& K(Y)= \begin{cases}1 & \text { if } 0<Y<Y_{i}=L_{f} / 2 L_{e} \\
k_{s} / k_{f} & \text { if } Y_{i}<Y<1\end{cases}
\end{aligned}
$$

where $f(Z, Y)$ and $f_{\text {ad }}(Z, Y)$ are preferably estimates of the steady-state solution or even any reasonable function, since in the present work we are only interested in the steady-state results. In order to solve problem (3), we define the following integral transform pairs for the heat exchange section,

$$
\begin{gathered}
\bar{\theta}_{i}(Z, t)=\int_{0}^{1} \tilde{\xi}_{i}(Y) \theta(Y, Z, t) d Y, \quad \text { transform } \\
\theta(Y, Z, t)=\sum_{i=1}^{\infty} \tilde{\xi}_{i}(Y) \bar{\theta}_{i}(Z, t), \quad \text { inverse }
\end{gathered}
$$

and for the upstream region:

$$
\begin{array}{cl}
\bar{\theta}_{\mathrm{ad}, \mathrm{i}}(Z, t)=\int_{0}^{1} \tilde{\zeta}_{i}(Y) \theta_{\mathrm{ad}}(Y, Z, t) d Y, & \text { transform } \\
\theta_{\mathrm{ad}}(Y, Z, t)=\sum_{i=1}^{\infty} \tilde{\zeta}_{i}(Y) \bar{\theta}_{\mathrm{ad}, \mathrm{i}}(Z, t), & \text { inverse }
\end{array}
$$

where the eigenfunctions $\xi_{i}(Y)$ and associated eigenvalues $\beta_{i}$, corresponding to the heat exchange section, come from the following eigenvalue problem:

$$
\begin{aligned}
& \frac{4}{\sigma^{2}} \frac{d}{d Y}\left(K(Y) \frac{d \xi_{i}(Y)}{d Y}\right)+\beta_{i}^{2} \xi_{i}(Y)=0 \\
& \left.\frac{d \xi_{i}}{d Y}\right|_{Y=0}=0,\left.\quad \frac{d \xi_{i}}{d Y}\right|_{Y=1}+\operatorname{Bi} \xi_{i}(1)=0
\end{aligned}
$$

while the eigenfunctions $\zeta_{i}(Y)$ and associated eigenvalues $\alpha_{i}$, corresponding to the upstream region, come from:

$$
\begin{gathered}
\frac{4}{\sigma^{2}} \frac{d}{d Y}\left(K(Y) \frac{d \zeta_{i}(Y)}{d Y}\right)+\alpha_{i}^{2} \zeta_{i}(Y)=0 \\
\left.\frac{d \zeta_{i}}{d Y}\right|_{Y=0}=0,\left.\quad \frac{d \zeta_{i}}{d Y}\right|_{Y=1}=0
\end{gathered}
$$


The normalized eigenfunctions, $\tilde{\xi}_{i}(Y)$ and $\tilde{\zeta}_{i}(Y)$ are calculated as:

$$
\tilde{\xi}_{i}(Y)=\frac{\xi_{i}(Y)}{\sqrt{N_{\xi_{i}}}} \quad \text { and } \quad \tilde{\zeta}_{i}(Y)=\frac{\zeta_{i}(Y)}{\sqrt{N_{\zeta_{i}}}}
$$

with the normalization integrals given by

$$
N_{\xi_{i}}=\int_{0}^{1} \xi_{i}^{2} d Y \quad \text { and } \quad N_{\zeta_{i}}=\int_{0}^{1} \zeta_{i}^{2} d Y
$$

The eigenvalue problems (6) and (7) are handled by the GITT itself, with the proposition of a simpler auxiliary eigenvalue problem and expanding the unknown eigenfunctions in terms of the chosen basis. Here we chose the simplest auxiliary problem keeping the boundary conditions of the original problem, which for the heat exchange section is written as

$$
\begin{gathered}
\frac{d^{2} \Omega_{n}(Y)}{d Y^{2}}+\lambda_{n}^{2} \Omega_{n}(Y)=0 \\
\left.\frac{d \Omega_{n}(Y)}{d Y}\right|_{Y=0}=0,\left.\quad \frac{d \Omega_{n}}{d Y}\right|_{Y=1}+\operatorname{Bi} \Omega_{n}(1)=0
\end{gathered}
$$

and for the upstream region is written as

$$
\begin{gathered}
\frac{d^{2} \phi_{n}(Y)}{d Y^{2}}+v_{n}^{2} \phi_{n}(Y)=0 \\
\left.\frac{d \phi_{n}(Y)}{d Y}\right|_{Y=0}=0,\left.\quad \frac{d \phi_{n}(Y)}{d Y}\right|_{Y=1}=0
\end{gathered}
$$

The proposed expansions of the original eigenfunctions are then given by

$$
\begin{gathered}
\xi_{i}(Y)=\sum_{n=1}^{\infty} \tilde{\Omega}_{n}(Y) \bar{\xi}_{i, n} \quad \text { inverse } \\
\bar{\xi}_{i, n}=\int_{0}^{1} \xi_{i}(Y) \tilde{\Omega}_{n}(Y) d Y \quad \text { transform }
\end{gathered}
$$

and

$$
\begin{gathered}
\zeta_{i}(Y)=\sum_{n=0}^{\infty} \tilde{\phi}_{n}(Y) \bar{\zeta}_{i, n} \quad \text { inverse } \\
\bar{\zeta}_{i, n}=\int_{0}^{1} \zeta_{i}(Y) \tilde{\phi}_{n}(Y) d Y \quad \text { transform }
\end{gathered}
$$


where the normalized eigenfunctions $\tilde{\Omega}_{n}(Y)$ and $\tilde{\phi}_{n}(Y)$ are given by the following expressions:

$$
\tilde{\Omega}_{n}(Y)=\frac{\Omega_{n}(Y)}{\sqrt{N_{\Omega_{n}}}} \quad \text { and } \quad \tilde{\phi}_{n}(Y)=\frac{\phi_{n}(Y)}{\sqrt{N_{\phi_{n}}}}
$$

with the normalization integrals given by

$$
N_{\Omega_{n}}=\int_{0}^{1} \Omega_{n}^{2} d Y \quad \text { and } \quad N_{\phi_{n}}=\int_{0}^{1} \phi_{n}^{2} d Y
$$

The integral transformation of the eigenvalue problems with space-variable coefficients, problems (6) and (7), is then performed by operating on Eq. (6a) with $\int_{0}^{1} \tilde{\Omega}_{n}(Y)-d Y$ and on Eq. (7a) with $\int_{0}^{1} \tilde{\phi}_{n}(Y)-d Y$, respectively [22, 23]. For problem (6), we then have

$$
\begin{gathered}
(\mathbf{A}-\nu \mathbf{B}) \bar{\xi}=0 \quad \text { with } \quad \nu_{i}=\beta_{i}^{2} \\
\bar{\xi}=\left\{\bar{\xi}_{n, m}\right\}, \quad \mathbf{B}=\left\{B_{n, m}\right\} \\
B_{n, m}=\int_{0}^{1} U(Y) \tilde{\Omega}_{n}(Y) \tilde{\Omega}_{m}(Y) d Y
\end{gathered}
$$

$$
\mathbf{A}=\left\{A_{n, m}\right\}
$$

$$
\begin{aligned}
A_{n, m} & =\int_{0}^{1} \tilde{\mathbf{\Omega}}_{m}(Y) \frac{4}{\sigma^{2}} \frac{d}{d Y}\left[K(Y) \frac{d \tilde{\mathbf{\Omega}}_{n}(Y)}{d Y}\right] d Y \\
& =\left.\frac{4}{\sigma^{2}} K(1) \tilde{\boldsymbol{\Omega}}_{m}(1) \frac{d \tilde{\mathbf{\Omega}}_{n}(Y)}{d Y}\right|_{Y=1}-\frac{4}{\sigma^{2}} \int_{0}^{1} K(Y) \frac{d \tilde{\mathbf{\Omega}}_{m}(Y)}{d Y} \frac{d \tilde{\boldsymbol{\Omega}}_{n}(Y)}{d Y} d Y
\end{aligned}
$$

while for problem (7) we have

$$
\begin{gathered}
(\mathbf{A}-\nu \mathbf{B}) \bar{\zeta}=0 \quad \text { with } \quad \nu_{i}=\alpha_{i}^{2} \\
\bar{\zeta}=\left\{\bar{\zeta}_{n, m}\right\}, \quad \mathbf{B}=\left\{B_{n, m}\right\} \\
B_{n, m}=\int_{0}^{1} U(Y) \tilde{\phi}_{n}(Y) \tilde{\phi}_{m}(Y) d Y \\
\mathbf{A}=\left\{A_{n, m}\right\} \\
A_{n, m}=\int_{0}^{1} \tilde{\phi}_{m}(Y) \frac{4}{\sigma^{2}} \frac{d}{d Y}\left[K(Y) \frac{d \tilde{\phi}_{n}(Y)}{d Y}\right] d Y \\
=-\frac{4}{\sigma^{2}} \int_{0}^{1} K(Y) \frac{d \tilde{\phi}_{m}(Y)}{d Y} \frac{d \tilde{\phi}_{n}(Y)}{d Y} d Y
\end{gathered}
$$

The algebraic problems (15a) and (16a) can be solved numerically to provide results for the eigenvalues and eigenvectors, upon truncation to a sufficiently large 
finite order $M$, and then combined by the inverse formulas $(12 a)$ and (13a) to provide the desired original eigenfunctions $\xi_{i}(Y)$ and $\zeta_{i}(Y)$, respectively.

One way to solve the coupled equations given in problem (3) would be to perform the integral transformation by means of Eqs. (4) and (5), yielding a transformed partial differential equation (PDE) system on $\bar{\theta}_{i}(Z, t)$ and $\bar{\theta}_{\mathrm{ad}, \mathrm{i}}(Z, t)$ coupled at the interface $Z=0$ [11]. Instead, we here choose to reformulate problem (3) assuming that the transition from the upstream region to the heat exchange section, i.e., the transition from the insulated to the convective boundary condition at $Y=1$, occurs abruptly, similarly to what has been proposed in the transverse direction, thus making use of the single-domain formulation also in the longitudinal direction. Therefore, we have

$$
\begin{gathered}
\frac{\partial \theta^{*}(Y, Z, t)}{\partial t}=-U(Y) \frac{\partial \theta^{*}}{\partial Z}+\frac{K(Y)}{\mathrm{Pe}^{2}} \frac{\partial^{2} \theta^{*}}{\partial Z^{2}}+\frac{4}{\sigma^{2}} \frac{\partial}{\partial Y}\left[K(Y) \frac{\partial \theta^{*}}{\partial Y}\right] \quad(17 a) \\
0<Y<1, \quad-Z_{\mathrm{ad}, \infty}<Z<Z_{\infty}, t>0 \\
\theta^{*}\left(Y,-Z_{\mathrm{ad}, \infty}, t\right)=1,\left.\quad \frac{\partial \theta^{*}}{\partial Z}\right|_{Z=Z_{\infty}}=0, \quad 0<Y<1, t>0 \\
\left.\frac{\partial \theta^{*}}{\partial Y}\right|_{Y=0}=0,\left.\quad \frac{\partial \theta^{*}}{\partial Y}\right|_{Y=1}+\operatorname{Bi}_{\mathrm{ef}}(Z) \theta^{*}(1, Z, t)=0, \quad-Z_{\mathrm{ad}, \infty}<Z<Z_{\infty}, t>0 \quad(17 b, c) \\
\theta^{*}(Y, Z, 0)=f_{\mathrm{ef}}(Z, Y), \quad 0<Y<1, \quad-Z_{\mathrm{ad}, \infty}<Z<Z_{\infty}
\end{gathered}
$$

with

$\mathrm{Bi}_{\mathrm{ef}}(Z)=\left\{\begin{array}{ll}0 & \text { if }-Z_{\mathrm{ad}, \infty}<Z<0 \\ \mathrm{Bi} & \text { if } 0<Z<Z_{\infty}\end{array}, f_{\mathrm{ef}}(Z, Y)= \begin{cases}f_{\mathrm{ad}}(Z, Y), & \text { if }-Z_{\mathrm{ad}, \infty}<Z<0 \\ f(Z, Y), & \text { if } 0<Z<Z_{\infty}\end{cases}\right.$

In this case, we may propose the following integral transform pair:

$$
\begin{array}{cl}
\bar{\theta}_{i}^{*}(Z, t)=\int_{0}^{1} \tilde{\psi}_{i}(Y, Z) \theta^{*}(Y, Z, t) d Y & \text { transform } \\
\theta^{*}(Y, Z, t)=\sum_{i=1}^{\infty} \tilde{\psi}_{i}(Y, Z) \bar{\theta}_{i}^{*}(Z, t) & \text { inverse }
\end{array}
$$

where the eigenfunctions $\psi(Y, Z)$ and corresponding eigenvalues $\mu(Z)$ are represented, respectively, by $\zeta(Y)$ and $\alpha$, for $-Z_{\mathrm{ad}, \infty} \leq Z \leq 0$, and by $\xi(Y)$ and $\beta$, for $0 \leq Z \leq Z_{\infty}$. Thus, we may write

$$
\begin{gathered}
\tilde{\psi}_{i}(Y, Z)=\tilde{\xi}_{i}(Y)+\left[\tilde{\zeta}_{i}(Y)-\tilde{\xi}_{i}(Y)\right] \delta(Z) \\
\mu_{i}(Z)=\beta_{i}+\left(\alpha_{i}-\beta_{i}\right) \delta(Z)
\end{gathered}
$$


where

$$
\delta(Z)=\frac{1}{1+e^{\eta Z}}
$$

where the parameter $\eta$ controls the transition spatial behavior. Figure 2 illustrates the transition behavior for four different values of $\eta$.

Then, operating Eq. $(17 a)$ with $\int_{0}^{1} \tilde{\psi}_{i}(Y, Z)-d Y$ and making use of the boundary conditions, we obtain the following PDE system:

$$
\frac{\partial \bar{\theta}_{i}^{*}(Z, t)}{\partial t}+\mu_{i}^{2}(Z) \bar{\theta}_{i}^{*}=g\left(Z, t, \bar{\theta}_{j}^{*}\right) \quad i, j=1,2, \ldots
$$

where

$$
g\left(Z, t, \bar{\theta}_{j}^{*}\right)=-\sum_{k=1}^{\infty} \frac{\partial \bar{\theta}_{i}^{*}}{\partial Z} \int_{0}^{1} U(Y) \tilde{\psi}_{i} \tilde{\psi}_{k} d Y-\sum_{k=1}^{\infty} \frac{\partial^{2} \bar{\theta}_{i}^{*}}{\partial Z^{2}} \int_{0}^{1} \frac{K(Y)}{\mathrm{Pe}^{2}} \tilde{\psi}_{i} \tilde{\psi}_{k} d Y
$$

with the transformed boundary and initial conditions given by

$$
\begin{aligned}
\bar{\theta}_{i}^{*}\left(-Z_{\mathrm{ad}, \infty}, t\right) & =\left.\int_{0}^{1} \tilde{\psi}_{i} d Y \quad \frac{\partial \bar{\theta}_{i}^{*}}{\partial Z}\right|_{Z=Z_{\infty}}=0 \\
\bar{\theta}_{i}^{*}(Z, 0) & =\int_{0}^{1} \tilde{\psi}_{i} f_{e f}(Z, Y) d Y
\end{aligned}
$$

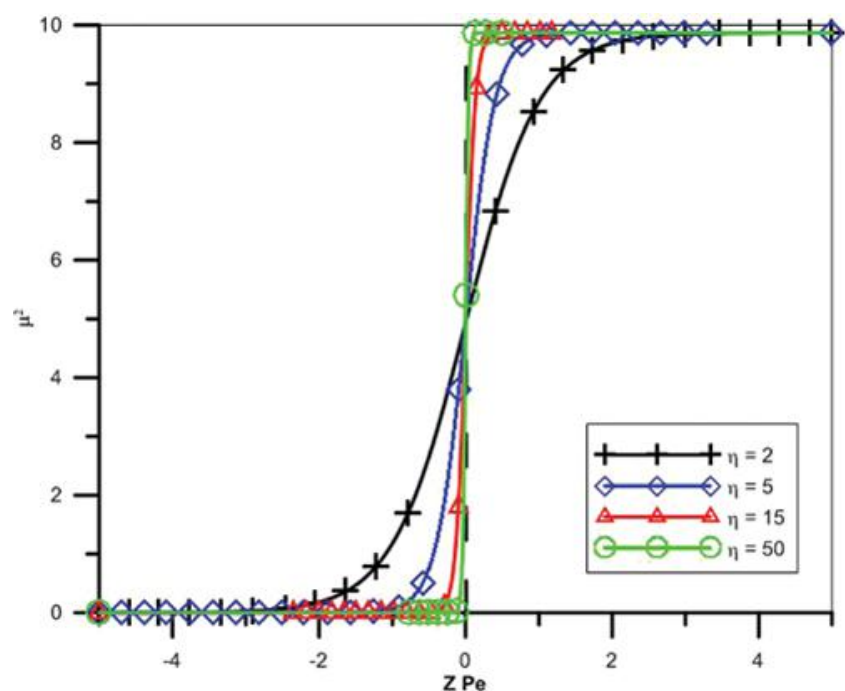

Figure 2. Influence of the parameter $\eta$ on the transition sharpness between two squared eigenvalues: $\beta_{1}^{2}=0$ and $\alpha_{1}^{2}=9.87 . \eta=2, \eta=5, \eta=15, \eta=50$ (color figure available online). 
System (20), after truncation to a sufficiently large order $N$, can be solved numerically to provide results for the transformed temperatures, $\bar{\theta}_{i}^{*}(Z, t)$. The Mathematica software package [25] provides the routine NDSolve for the solution of the PDE system considered here, under automatic absolute and relative error control. Once the transformed potentials have been computed numerically, the Mathematica routine automatically provides an interpolating function object that approximates the $Z$ and $t$ variables behavior of the solution in a continuous form. Then, the inversion formula $(18 b)$ can be recalled to yield the potential field representation $\theta^{*}$ at any desired position $(Y, Z)$ and time $t$. However, in this work we are only interested in the steady-state solution, after passing the pseudo-transient period.

The main interest in convective heat transfer analysis is often to determine local Nusselt numbers. The following expressions for the local Nusselt number and for the bulk temperature are then here employed:

$$
\mathrm{Nu}(Z)=-\left.\frac{1}{\theta_{\text {bulk }}(Z)-\theta\left(Y_{i}, Z\right)} \frac{\partial \theta(Y, Z)}{\partial Y}\right|_{Y=Y_{i}}, \quad \theta_{\text {bulk }}(Z)=\frac{\int_{0}^{Y_{i}} U(Y) \theta(Y, Z) d Y}{\int_{0}^{Y_{i}} U(Y) d Y}
$$

In order to avoid the direct evaluation of the derivative $\partial \theta /\left.\partial Y\right|_{Y=Y_{i}}$ at the interface, the following alternative integral balance formula [21] has been used:

$$
\int_{0}^{Y_{i}} U(Y) \frac{\partial \theta}{\partial Z} d Y=\int_{0}^{Y_{i}} \frac{K(Y)}{\mathrm{Pe}^{2}} \frac{\partial^{2} \theta}{\partial Z^{2}} d Y+\int_{0}^{Y_{i}} \frac{4}{\sigma^{2}} \frac{\partial}{\partial Y}\left[K(Y) \frac{\partial \theta}{\partial Y}\right] d Y
$$

yielding

$$
\left.\frac{\partial \theta}{\partial Y}\right|_{Y=Y_{i}}=\frac{\sigma^{2}}{4}\left[\int_{0}^{Y_{i}} U(Y) \frac{\partial \theta}{\partial Z} d Y-\int_{0}^{Y_{i}} \frac{K(Y)}{\mathrm{Pe}^{2}} \frac{\partial^{2} \theta}{\partial Z^{2}} d Y\right]
$$

Once the inverse formula, Eq. (4b), is substituted into Eq. (22b) above and the corresponding integral coefficients are determined analytically, an alternative expansion with improved convergence characteristics is obtained to evaluate the heat flux at the fluid-solid interface, and consequently the local Nusselt number.

\section{RESULTS AND DISCUSSION}

The proposed model taking into account the coupling of the heat transfer section with the upstream region, $-Z_{\mathrm{ad}, \infty}<Z<0$, including the single-domain reformulation in the longitudinal direction and together with its associated solution methodology, are first critically compared with a simpler version of this problem, studied before by Castellões et al. [11]. In that work the internal convection problem had been tackled by the traditional approach, separately handling the two longitudinal domains coupled by continuity conditions at the interface $Z=0$. This verification problem is a special case of problem (1) above (Figure 1), with the channel walls assumed to be thermally thin, so that their participation in the heat transfer problem can be neglected. Also, in that simpler problem [11], instead 
of convective boundary condition at the walls, we have a prescribed temperature condition.

Figure 3 shows the fluid bulk temperature evolution in this special case, as obtained by means of the single-domain approach presented in this work, for different values of the parameter $\eta$ which controls the sharpness of the transition between the two domains in the longitudinal direction. The problem under consideration has $\mathrm{Pe}=1$, and the figure depicts the evolution of the fluid bulk temperature along the channel length, with $\eta=1, \eta=2, \eta=5$, and $\eta=15$. One may observe that the curves for $\eta=5$ and $\eta=15$ are already essentially coincident to the graph scale and very little would be changed by considering a higher value of the spatial behavior parameter. In all such results, full convergence to four significant digits has been obtained in terms of the truncation order of the eigenfunction expansion in Eq. (18b), achieved with $N=10$ terms.

Completing the initial verifications on the solution obtained via the single-domain approach in the longitudinal direction, Figure 4 reports the comparison with the solution previously obtained in [11], which was obtained by means of the traditional approach, where separated problems are formulated in the two domains, the upstream region, $-Z_{\mathrm{ad}, \infty}<Z<0$, and the heat exchange section, $0<Z<Z_{\infty}$, coupled at the interface, $Z=0$, by means of continuity conditions. It should be recalled that the solution in [11] had been previously verified against the analytical results obtained by Tan and Normandia [26]. Figure 4 shows results for the fluid bulk temperature with three different Péclet numbers, $\mathrm{Pe}=1, \mathrm{Pe}=10$, and $\mathrm{Pe}=30$, covering a typical range for which heat transfer to the upstream region may be important in this problem, and excellent agreement between the two GITT solutions is observed, thus verifying the solution obtained via the single-domain approach.

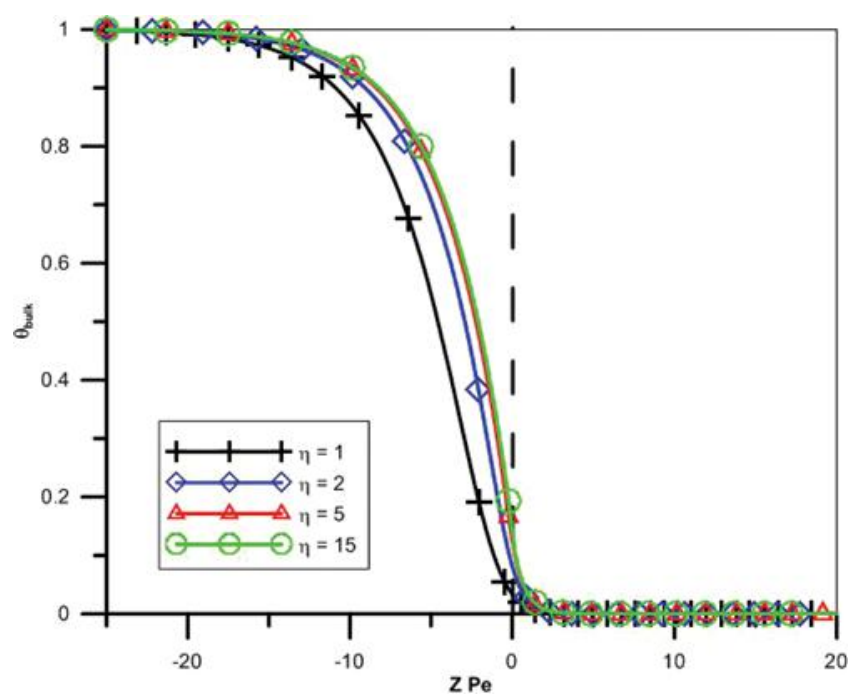

Figure 3. Convergence of the solution for the fluid bulk temperature via the single-domain approach in the longitudinal direction with respect to the parameter $\eta$, Eqs. $(19 a-c)$, where $\eta=1, \eta=2, \eta=5$, and $\eta=15$ (color figure available online). 


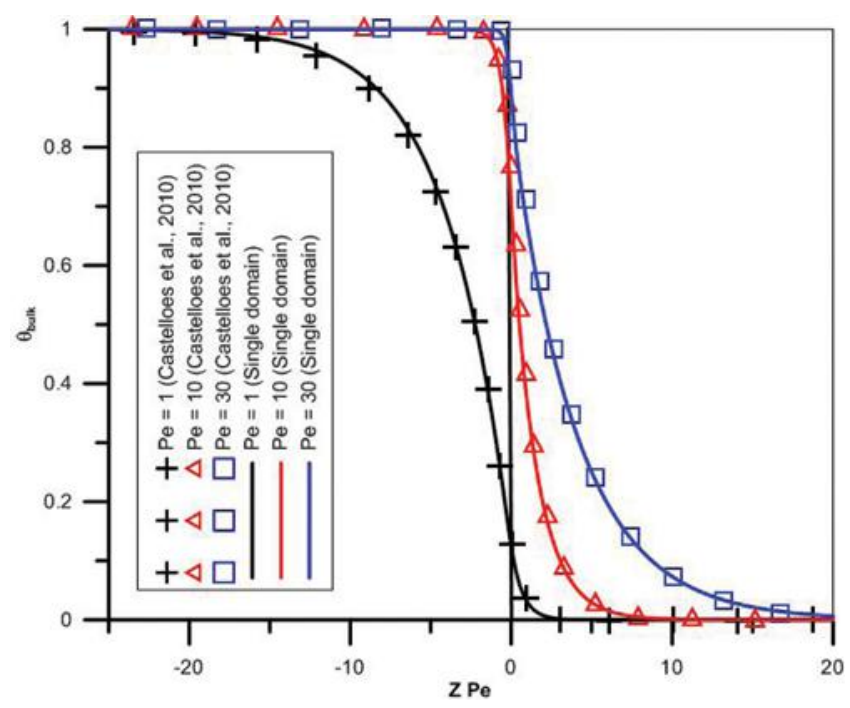

Figure 4. Comparison of the integral transform solution obtained via the single-domain approach in the longitudinal direction with that of Castellões et al. [11], for $\mathrm{Pe}=1, \mathrm{Pe}=10$, and $\mathrm{Pe}=30$ (color figure available online).

Now, we proceed to the full version of problem (1), considering the participation of the channel walls in the heat transfer problem through both transverse and axial heat conduction. The dimensionless thermophysical properties have been calculated motivated by an application with a microchannel manufactured on a nanocomposite material [22], made of polyester resin filled with aluminum oxide nanoparticles to obtain enhanced thermal conductivity $\left(k_{s}=0.204 \mathrm{~W} / \mathrm{m}^{\circ} C\right)$ with water as the working fluid $\left(k_{f}=0.64 \mathrm{~W} / \mathrm{m}^{\circ} C\right)$, so that $k_{s} / k_{f}=0.32$. We thus have a rectangular microchannel with height $L_{f}=200 \mu \mathrm{m}$, width $L_{w}=4 \mathrm{~cm}$, and wall thickness $300 \mu \mathrm{m}$. We consider three different prescribed volumetric flow rates, $\nu=0.126 \mathrm{~mL} / \mathrm{min}, \nu=0.252 \mathrm{~mL} / \mathrm{min}$, and $\nu=0.504 \mathrm{~mL} / \mathrm{min}$, yielding $P e=0.75$, $\mathrm{Pe}=1.5$, and $\mathrm{Pe}=3.0$, respectively. The microchannel external wall is assumed to be submitted to forced convection with air, yielding $\mathrm{Bi}=0.11\left(h_{e}=60 \mathrm{~W} / \mathrm{m}^{2 \circ} \mathrm{C}\right)$, in Eq. (3c). Figures $5 a$ and $5 b$ illustrate the actual behavior of the space-variable coefficients that are feeding the single-region model in problem (15), $U(Y)$ and $K(Y)$, as space-variable functions where the region from $Y=0$ to $Y_{i}=0.25$ corresponds to the fluid flow domain and the region from $Y=Y_{i}=0.25$ to 1 corresponds to the microchannel wall.

We first investigated the solution of the eigenvalue problems (6) and (7). Table 1 shows the first 10 eigenvalues calculated for different truncation orders $M$ in the expansion in Eq. (12a), problem (6), where we observe excellent convergence behavior to at least the fourth significant digit for $M<50$. Figure 6 depicts the convergence behavior of the 15 th eigenfunction in problem (6) with $\mathrm{Pe}=0.75, \xi_{15}(Y)$, where it can be confirmed that with $M=35$ terms the eigenfunction is already fully converged to the graph scale. Similar behavior is observed for the solution of eigenvalue problem (7), which is here omitted for the sake of brevity. 


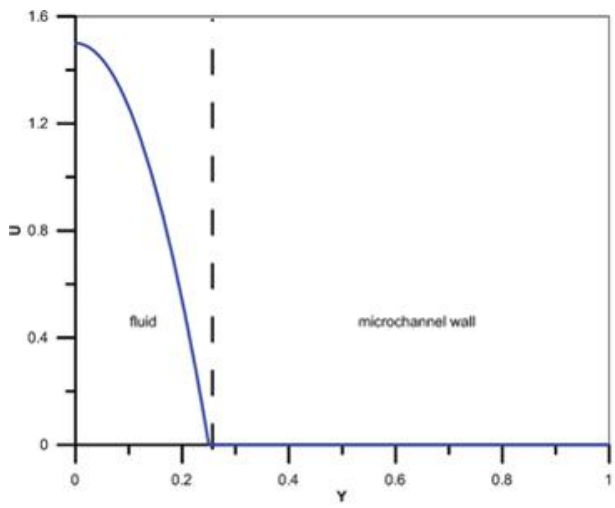

(a)

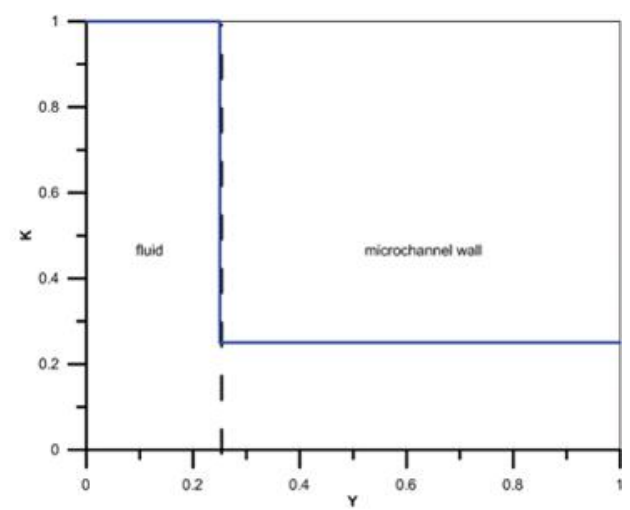

(b)

Figure 5. Representation of the space-variable coefficients as functions with the abrupt transition occurring at the interface fluid-solid wall: $(a) U(Y)$ and $(b) K(Y)$ (color figure available online).

Also, in order to give clear insight into the influence of the truncation order in the eigenvalue problems solution expansions, $M$, on the calculated temperatures, Table 2 shows the convergence behavior of the bulk temperatures for the first case considered in this work $(\mathrm{Pe}=0.75)$. In all situations, $N=15$ terms has been used in the final solution expansion, in Eq. (18b), yielding full convergence to the five digits shown. One may observe in Table 2 that the calculated temperatures at the selected positions are converged to at least the fourth significant digit for $M<50$.

Before proceeding to the discussion of the thermal behavior, a critical comparison is undertaken between the solution obtained by means of GITT with the single-domain model and the purely numeric solution obtained through the commercial solver COMSOL Multiphysics [27], employing physics-controlled automatic mesh generation under the extremely fine option. This verification has been carried out for $\mathrm{Pe}=0.75, \mathrm{Pe}=1.5$, and $\mathrm{Pe}=3$, and the results are depicted in Figure 7, where one may observe that the two solutions are essentially coincident once the mesh refinement option in the numerical solver is invoked.

Figures 8, 9, and 10 present the solutions for the three volumetric flow rates considered here, yielding $\mathrm{Pe}=0.75, \mathrm{Pe}=1.5$, and $\mathrm{Pe}=3.0$, respectively. In these

Table 1. Convergence behavior of the first 10 eigenvalues in problem (6)

\begin{tabular}{rlllll}
\hline$\beta_{i}$ & $M=30$ & $M=35$ & $M=40$ & $M=45$ & $M=50$ \\
\hline 1 & 0.386248 & 0.386242 & 0.386239 & 0.386236 & 0.386235 \\
2 & 1.74052 & 1.74001 & 1.73964 & 1.73943 & 1.73925 \\
3 & 3.49211 & 3.49 & 3.48847 & 3.48759 & 3.48685 \\
4 & 5.38427 & 5.38033 & 5.37745 & 5.3758 & 5.37443 \\
5 & 7.31265 & 7.3073 & 7.30334 & 7.30108 & 7.29924 \\
6 & 9.20838 & 9.20294 & 9.1988 & 9.19645 & 9.19457 \\
7 & 10.9806 & 10.9781 & 10.9759 & 10.9746 & 10.9737 \\
8 & 12.5827 & 12.5821 & 12.5819 & 12.5817 & 12.5816 \\
9 & 14.2323 & 14.2259 & 14.2222 & 14.2201 & 14.2181 \\
10 & 16.0617 & 16.0489 & 16.0407 & 16.0361 & 16.0321 \\
\hline
\end{tabular}




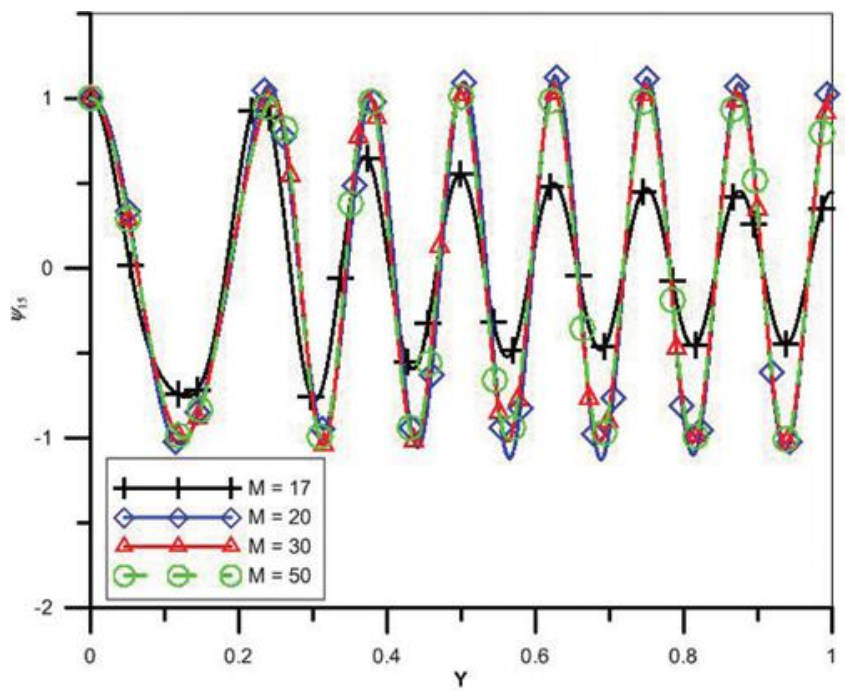

Figure 6. Convergence behavior of the 15 th eigenfunction of problem (6) with $\mathrm{Pe}=0.75$, for different truncation orders in Eq. (12a), $M=15, M=35$, and $M=50$ (color figure available online).

results we present, respectively, $(a)$ the evolution of the fluid bulk temperature along the microchannel length until $Z \mathrm{Pe}=50,75$, and 100 , which correspond to the dimensional values $z=2 \mathrm{~cm}, z=3 \mathrm{~cm}$, and $z=4 \mathrm{~cm} ;(b)$ the fluid temperature at the microchannel internal wall, $\theta_{\text {int }}(Z)$, from $Z \mathrm{Pe}=0$ to $Z \mathrm{Pe}=5$; and $(c)$ the evolution of the local Nusselt number, $\mathrm{Nu}(Z)$. Besides the solution of the full version of problem (1), i.e., including axial conduction in both the fluid and wall and taking into account the participation of the upstream region, we show the solution of two simplified formulations: considering the axial conduction but neglecting the participation of the upstream region [21], and completely neglecting the axial conduction term in both the wall and the fluid and the upstream region participation [14].

The results reveal that in this application with a microchannel etched on a nanocomposite substrate and low volumetric flow rates, neglecting the participation of the channel walls, the axial conduction in the fluid, and the participation of the upstream region may lead to significant deviations on the fluid bulk and wall temperatures, with the highest deviations being observed for the lowest Péclet

Table 2. Convergence of the calculated fluid bulk temperatures with respect to the truncation order in the eigenvalue problems solution expansion, $M$

\begin{tabular}{lcccc}
\hline$M$ & $Z \mathrm{Pe}=-10$ & $Z \mathrm{Pe}=-5$ & $Z \mathrm{Pe}=0$ & $Z \mathrm{Pe}=10$ \\
\hline$M=30$ & 0.99678 & 0.97354 & 0.78568 & 0.26944 \\
$M=35$ & 0.99679 & 0.97358 & 0.78625 & 0.26961 \\
$M=40$ & 0.99692 & 0.97468 & 0.79943 & 0.27306 \\
$M=45$ & 0.99691 & 0.97462 & 0.79878 & 0.27289 \\
$M=50$ & 0.99691 & 0.97463 & 0.79883 & 0.27291 \\
\hline
\end{tabular}




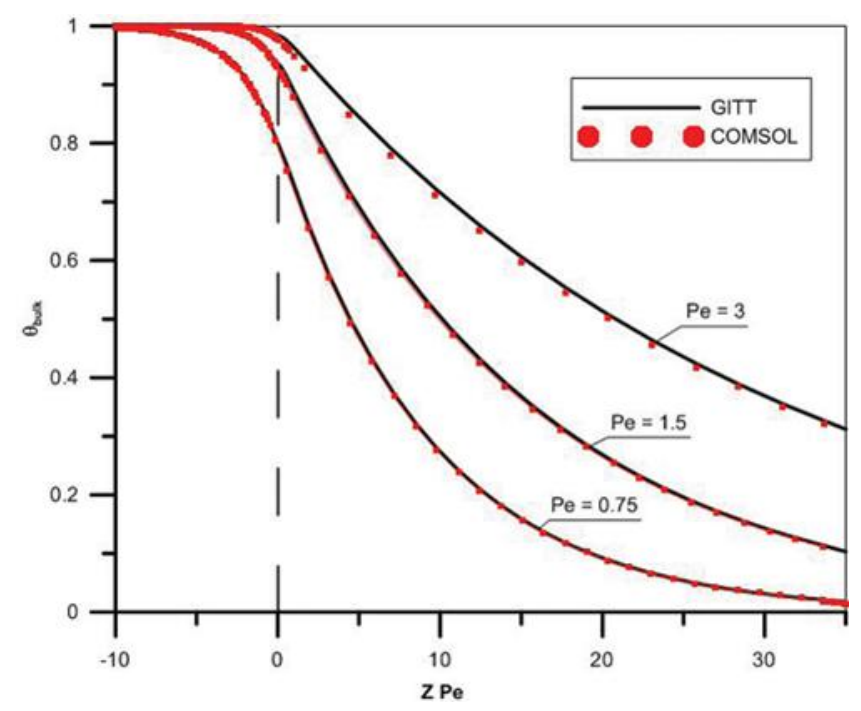

Figure 7. Co-validation between the solution obtained by means of GITT with the single-domain formulation and the numeric solution obtained through COMSOL Multiphysics (color figure available online).

numbers, as expected. It is noticeable that for the simplest model without axial diffusion, the transverse heat loss at each cross section is maximized, yielding the highest decay rate of the mean fluid temperature along the channel. When only forward axial heat diffusion is accounted for, the preheating effect is noticeable on the higher values of the fluid temperature at downstream axial positions. When backwards axial diffusion at the upstream region is also considered, it becomes clear that the precooling of the fluid takes place upstream of the heat exchange section entrance, thus affecting the actual inlet fluid temperature profile at the heat exchange section $(Z=0)$.

One may also observe that thermal development is achieved in a very short distance for the conjugated problem formulation that neglects the axial diffusion in both the fluid and wall regions, here represented by the red curves. However, it should be recalled that this model is not in fact applicable in the range of Péclet numbers considered here, since fluid axial diffusion is normally neglected only for Pe $>50$, but the results are shown here anyway, to provide an idea of the marked deviations encountered in such a poor modeling choice. Similar deviations could be encountered when using a correlation for the local Nusselt number, developed for a macroscale situation in which the effects of axial conduction in both the walls and the fluid could be neglected. When axial diffusion effects are introduced, since the transversal heat transfer evolution is now markedly modified, the thermal region development is also noticeably different, and clearly the development lengths become much larger than in the model when axial diffusion is neglected. As expected, the lower values of Péclet number lead to shorter thermal development lengths in both models when axial diffusion is incorporated into the formulation. At the lowest Péclet number the thermal development length is noticeably shorter when the upstream region takes part in the heat transfer process, anticipating the interactions 


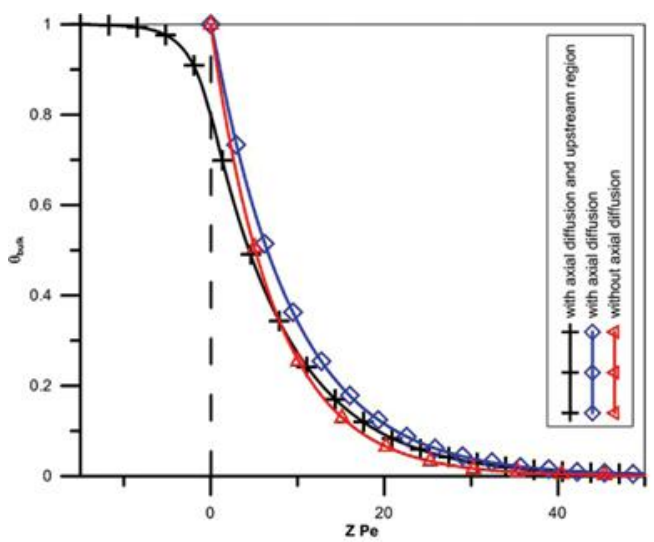

(a)

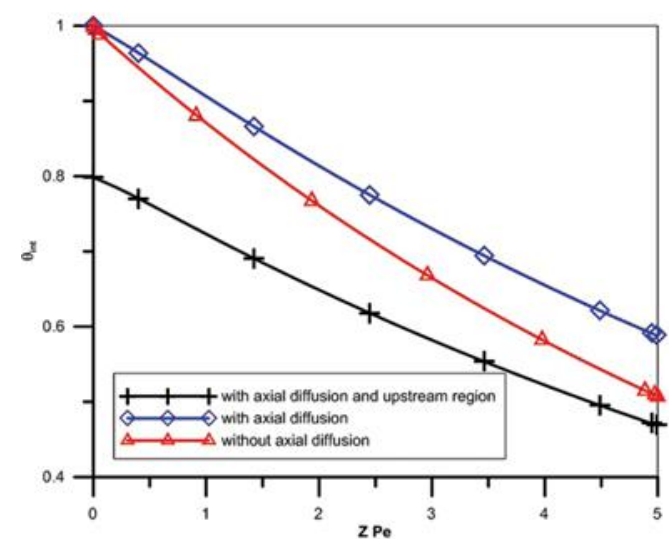

(b)

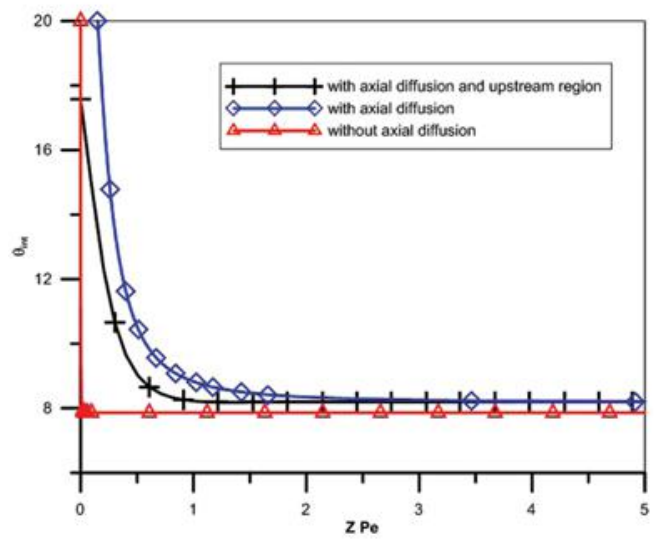

(c)

Figure 8. $\mathrm{Pe}=0.75$. (a) Fluid bulk temperature along the microchannel length. (b) Fluid temperature at the microchannel internal wall. (c) Local Nusselt number. Conjugated problem neglecting axial diffusion; considering axial diffusion but neglecting the upstream region; and considering axial diffusion and the upstream region (color figure available online). 


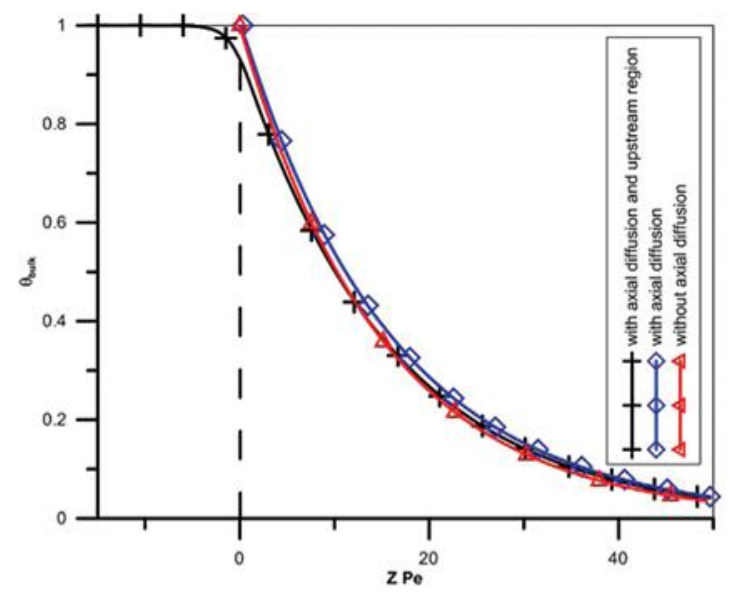

(a)

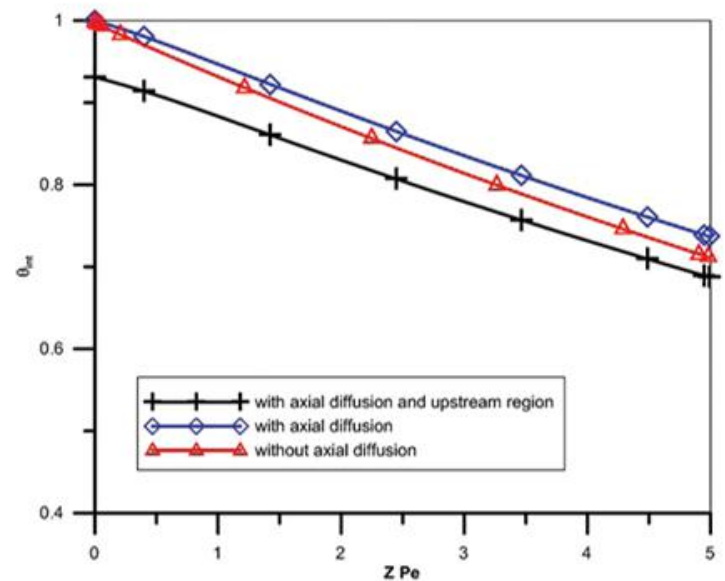

(b)

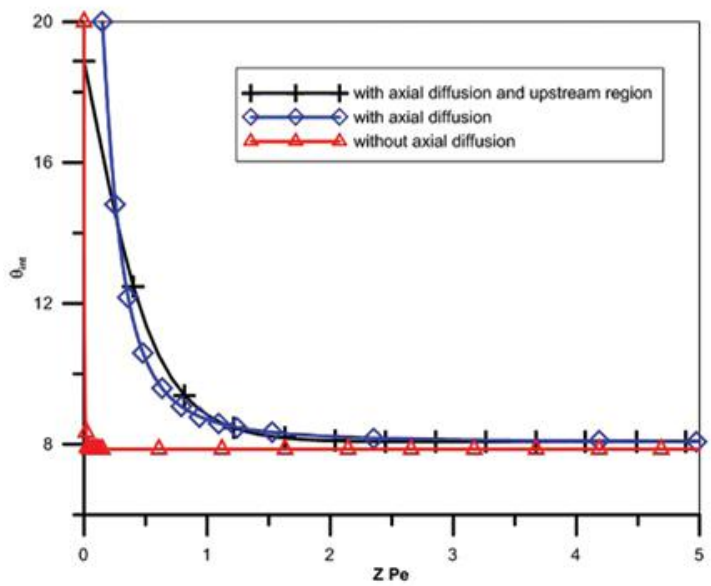

(c)

Figure 9. Same for $\mathrm{Pe}=1.5$ (color figure available online). 


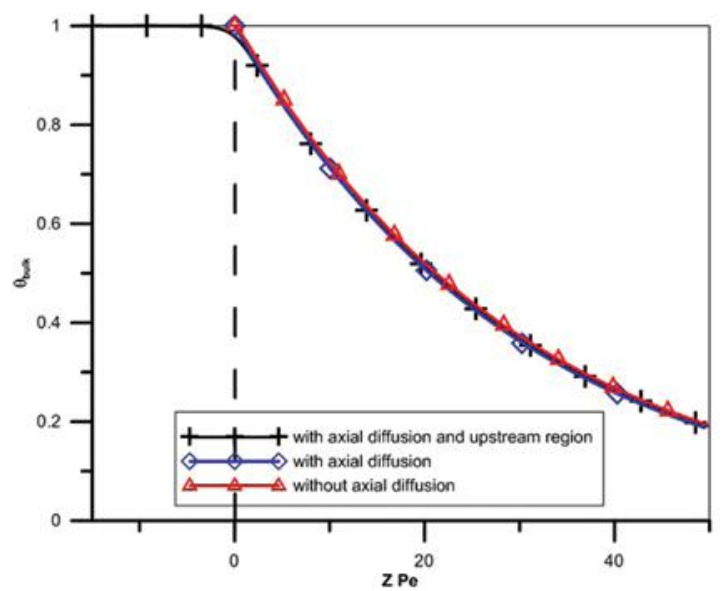

(a)

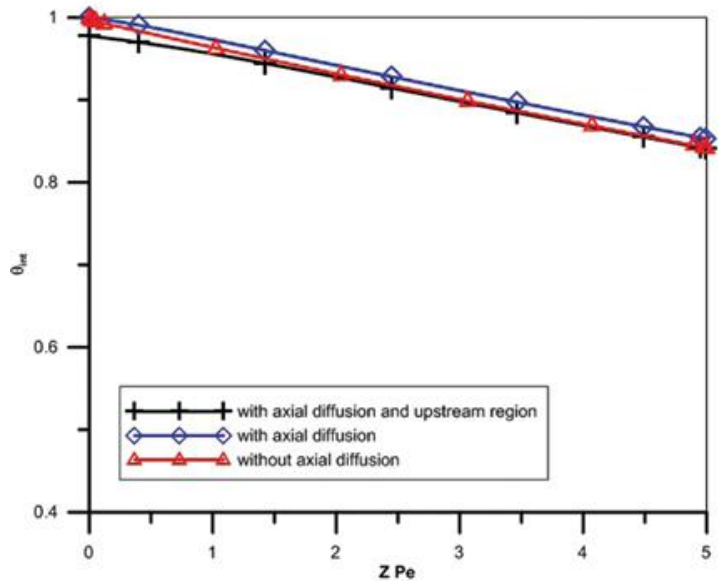

(b)

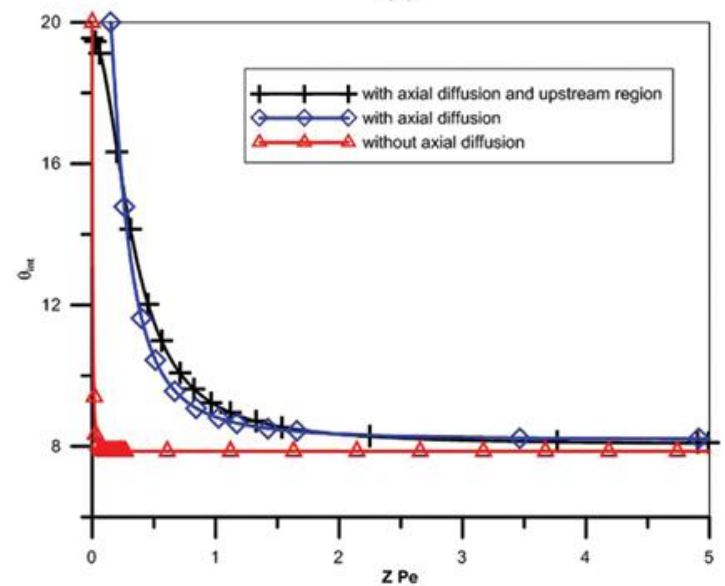

(c)

Figure 10. Same for $\mathrm{Pe}=3.0$ (color figure available online). 
between the fluid and the bounding walls. Then, marked differences in the local heat transfer coefficient can be achieved in the region closer to the entry even for these two models with axial diffusion effects.

Finally, Figures $11 a$ and $11 b$ show, respectively, the evolution of the fluid bulk temperatures and local Nusselt numbers along the channel length for $\mathrm{Pe}=30$, upto $Z \mathrm{Pe}=600$, corresponding to the dimensional value of $z=40 \mathrm{~cm}$. This case would correspond to a volumetric flow rate of $\nu=5.04 \mathrm{~mL} / \mathrm{min}$, which would be relatively high for the microchannel application considered here, but illustrates the progressive agreement of the solutions from the three different models. For such higher Péclet number, the three curves that show the fluid bulk temperatures in Figure $10 \mathrm{a}$ are essentially coincident. Moreover, in Figure $10 b$ we notice that the thermal

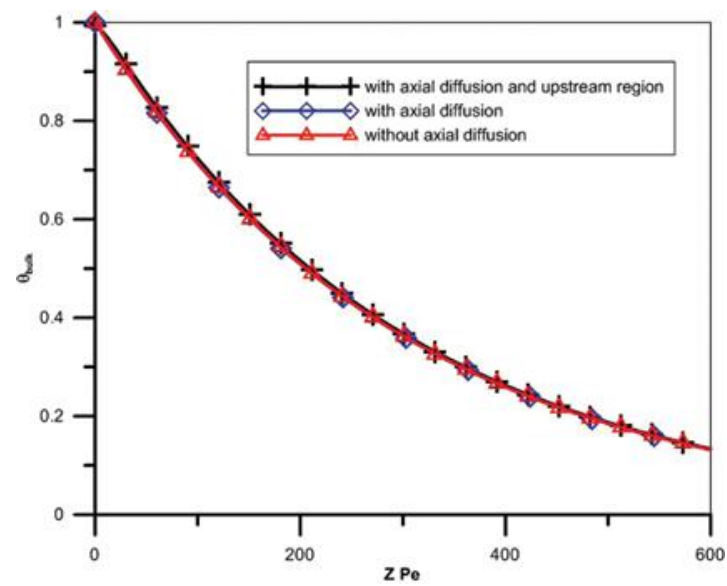

(a)

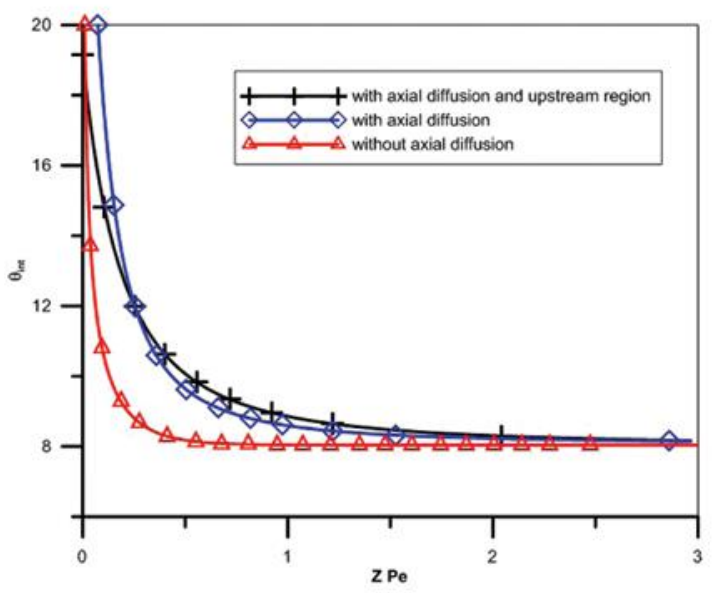

(b)

Figure 11. $\mathrm{Pe}=30$. (a) Fluid bulk temperature along the microchannel length. (b) Local Nusselt number. Conjugated problems neglecting axial diffusion in both the walls and the fluid; considering axial diffusion but neglecting the effect of the upstream region; considering axial diffusion and the participation of the upstream region (color figure available online). 
development length for the model neglecting the axial conduction term is now closer the other two models, while the asymptotic values of the Nusselt number are practically coincident for the three cases.

In order to investigate the behavior of the direct solution of the above single-domain formulation by a purely numerical method, we have attempted to solve the original partial differential problem through the NDSolve routine of the Mathematica package [25] under automatic accuracy control. However, under default specifications, it was not able to solve the problem accurately with the intrinsic maximum refinement options. We have also tried to impose limits of refinement higher than the default options, upto the maximum available computation capability, and still no satisfactory results were obtained. Apparently, only under a priori imposed variable meshing or adaptive meshing refinement can the fully discrete approach provide final results of closer accuracy to the present hybrid numericalanalytical approach. This observation on the robust and accurate performance of the hybrid method, comes from the fact that the problem coefficients with abrupt transitions are incorporated directly into the eigenvalue problem and the associated eigenfunctions carry relevant information on the spatial behavior of the solution. The results obtained in this work and recent related research [14, 21] suggest the feasibility of the single-domain formulation approach in dealing with conjugated heat transfer problems and, thus, the possibility of its implementation in combination hybrid analytical-numerical automatic solvers [24].

\section{CONCLUSIONS}

A single-domain formulation for conjugated convective-conductive heat transfer is further advanced by considering the multidomain original problem as a nonhomogeneous region with space-variable properties and parameters. The resulting unified formulation is handled by the generalized integral transform technique (GITT), which is employed both in the integral transformation of the combined solid-fluid energy equation and in the solution of the associated eigenvalue problem with variable coefficients. An application is considered related to flow within a microchannel fabricated on a nanocomposite polymeric substrate. Convergence of the proposed eigenfunction expansions are then illustrated and the solution is verified against previously reported analytical approaches. Particular attention is given to the analysis of the influence of the supposedly insulated upstream region on the heat transfer behavior of the fluid within the actual heat exchange section. At sufficiently low Péclet numbers, not only does axial diffusion at both the walls and fluid regions become essential to adequate modeling of the microscale conjugate problem, but also the precooling (or preheating) effect into the upstream region can markedly change the behavior of the local heat transfer coefficients in the thermal entry region of the heat exchange section. Within such a range, the use of Nusselt number correlations originally developed at the macroscale would lead to significantly erroneous predictions.

The extension of the present approach to three-dimensional problems essentially requires the formulation of two-dimensional eigenvalue problems, which can be readily tackled by the same methodology [17]. Although in principle an approximation, this approach has provided excellent results in the present conjugate heat 
transfer analysis, with user-prescribed accuracy once the spatial behavior of the abrupt transitions and the truncation orders of the related eigenfunction expansions are adequately controlled. In conclusion, this work adds to the available standard purely numerical simulation tools, either as a companion in co-validation tasks, or as an alternative approach for analytically oriented users, being especially attractive for implementation in automatic solvers.

\section{REFERENCES}

1. N. Kockmann, Transport Phenomena in Micro Process Engineering, Springer-Verlag, Berlin, 2008.

2. C. B. Sobhan and G. P. Peterson, Microscale and Nanoscale Heat Transfer: Fundamentals and Engineering Applications, CRC Press, Boca Raton, FL, 2008.

3. G. L. Morini, Single-Phase Convective Heat Transfer in Microchannels: A Review of Experimental Results, Int. J. Thermal Sci., vol. 43, pp. 631-651, 2004.

4. Y. Yener, S. Kakaç, M. Avelino, and T. Okutucu, Single-Phase Forced Convection in Microchannels-A State-of-the-Art Review, in S. Kakaç, L. L. Vasiliev, Y. Bayazitoglu, and Y. Yener (eds.), Microscale Heat Transfer-Fundamentals and Applications, NATO ASI Series, Kluwer Academic Publishers, Dordrecht, The Netherlands, pp. 1-24, 2005.

5. S. Yu and T. A. Ameel, Slip Flow Heat Transfer in Rectangular Microchannels, Int. J. Heat Mass Transfer, vol. 44, pp. 4225-4234, 2001.

6. G. Tunc and Y. Bayazitoglu, Heat Transfer in Microtubes with Viscous Dissipation, Int. J. Heat Mass Transfer, vol. 44, pp. 2395-2403, 2001.

7. G. Tunc and Y. Bayazitoglu, Heat Transfer in Rectangular Microchannels, Int. J. Heat Mass Transfer, vol. 45, pp. 765-773, 2002.

8. M. D. Mikhailov and R. M. Cotta, Mixed Symbolic-Numerical Computation of Convective Heat Transfer with Slip Flow in Microchannels, Int. Commun. Heat Mass Transfer, vol. 32, pp. 341-348, 2005.

9. R. M. Cotta, S. Kakaç, M. D. Mikhailov, F. V. Castellões, and C. R. Cardoso, Transient Flow, and Thermal Analysis in Microfluidics, in S. Kakaç, L. L. Vasiliev, Y. Bayazitoglu, and Y. Yener (eds.), Microscale Heat Transfer-Fundamentals and Applications, NATO ASI Series, Kluwer Academic Publishers, Dordrecht, The Netherlands, pp. 175-196, 2005.

10. F. V. Castellões, C. R. Cardoso, P. Couto, and R. M. Cotta, Transient Analysis of Slip Flow and Heat Transfer in Microchannels, Heat Transfer Eng., vol. 28, no. 6, pp. 549-558, 2007.

11. F. V. Castellões, J. N. N. Quaresma, and R. M. Cotta, Convective Heat Transfer Enhancement in Low Reynolds Number Flows with Wavy Walls, Int. J. Heat Mass Transfer, vol. 53, pp. 2022-2034, 2010.

12. G. Maranzana, I. Perry, and D. Maillet, Mini and Microchannels: Influence of Axial Conduction in the Walls, Int. J. Heat Mass Transfer, vol. 47, pp. 3993-4004, 2004.

13. J. S. Nunes, R. M. Cotta, M. Avelino, and S. Kakaç, Conjugated Heat Transfer in Microchannels, in S. Kakaç, B. Kosoy and A. Pramuanjaroenkij (eds.), Microfluidics Based Microsystems: Fundamentals and Applications, NATO Science for Peace and Security Series A: Chemistry and Biology, vol. 1, pp. 61-82, 2010.

14. D. C. Knupp, C. P. Naveira-Cotta, and R. M. Cotta, Theoretical Analysis of Conjugated Heat Transfer with a Single Domain Formulation and Integral Transforms, Int. Commun. Heat Mass Transfer, vol. 39, pp. 355-362, 2012.

15. R. M. Cotta, Hybrid Numerical-Analytical Approach to Nonlinear Diffusion Problems, Numer. Heat Transfer B, vol. 127, pp. 217-226, 1990.

16. R. M. Cotta, Integral Transforms in Computational Heat and Fluid Flow, CRC Press, Boca Raton, FL, 1993. 
17. R. M. Cotta, Benchmark Results in Computational Heat and Fluid Flow: The Integral Transform Method, Int. J. Heat Mass Transfer, vol. 37, suppl. 1, pp. 381-394, 1994.

18. R. M. Cotta and M. D. Mikhailov, Heat Conduction: Lumped Analysis, Integral Transforms, Symbolic Computation, Wiley-Interscience, Chichester, UK, 1997.

19. L. S. B. Alves and R. M. Cotta, Transient Natural Convection inside Porous Cavities: Hybrid Numerical-Analytical Solution and Mixed Symbolic-Numerical Computation, Numer. Heat Transfer A, vol. 38, pp. 89-110, 2000.

20. R. M. Cotta and M. D. Mikhailov, Hybrid Methods and Symbolic Computations, in W. J. Minkowycz, E. M. Sparrow and J. Y. Murthy (eds.), Handbook of Numerical Heat Transfer, 2nd ed., chap. 16, John Wiley, New York, 2006.

21. D. C. Knupp, C. P. Naveira-Cotta, and R. M. Cotta, Conjugated Heat Transfer in MicroChannels with a Single Domain Formulation and Integral Transforms, Paper MNHMT2012-75287, Proc. ASME 2012 3rd Micro/Nanoscale Heat \& Mass Transfer International Conference, Atlanta, GA, USA, March 3-6, 2012.

22. D. C. Knupp, C. P. Naveira-Cotta, J. V. C. Ayres, R. M. Cotta, and H. R. B. Orlande, Theoretical-Experimental Analysis of Heat Transfer in Non-homogeneous Solids via Improved Lumped Formulation, Integral Transforms and Infrared Thermography, Int. J. Thermal Sci., vol. 62, pp. 71-84, 2012.

23. C. P. Naveira-Cotta, H. R. B. Orlande, and R. M. Cotta, Integral Transforms, and Bayesian Inference in the Identification of Variable Thermal Conductivity in Two-Phase Dispersed Systems, Numer. Heat Transfer B, vol. 57, pp. 1-30, 2010.

24. R. M. Cotta, D. C. Knupp, C. P. Naveira-Cotta, L. A. Sphaier, and J. N. N. Quaresma, Unified Integral Transforms Algorithm for Solving Multidimensional Nonlinear Convection-Diffusion Problems, Numer. Heat Transfer A, vol. 63, pp. 1-27, 2013.

25. S. Wolfram, The Mathematica Book, Wolfram Media, Cambridge, 2005.

26. C. W. Tan and M. J. Normandia, Low Péclet Number Diffusion in Narrow Rectangular Channels, Lett. Heat Mass Transfer, vol. 2, pp. 259-266, 1975.

27. COMSOL Multiphysics, version 4.2, Multiphysics Modeling, Finite Element Analysis, and Engineering Simulation Software, EUA, 1998-2013. 